\title{
Monitoring the Decrease in Susceptibility to Ribosomal RNAs Targeting Antimicrobials and Its Molecular Basis in Clinical Mycoplasma bovis Isolates over Time
}

\author{
Dima Khalil,1,2 Claire A.M. Becker, 1,2 and Florence Tardy 1,2
}

Mycoplasma bovis is considered an emerging threat to bovine production in industrialized countries. Its control depends on good husbandry and efficient chemotherapy practices. In France, clinical isolates collected after 2009 showed a drastic loss of susceptibility to most antimicrobials when compared with isolates collected in 1978-1979. The aim of the present study was to analyze the molecular mechanisms underlying the shift toward resistance to macrolides and tetracyclines and to assess whether the clinical origin of the isolates or their molecular subtypes could have influenced their pattern of evolution. We demonstrated that all M. bovis isolates collected as early as 2000 should already be considered resistant to tylosin, tilmicosin, and oxytetracycline, whatever the associated clinical signs. The shift toward resistance happened earlier for oxytetracycline and more progressively for tylosin/tilmicosin. Isolates belonging to the major subtype ST2 $(n=40)$ showed a homogeneous genotype for resistance, with combined alterations of $\mathrm{G}_{748} \mathrm{~A}$ and $\mathrm{A}_{2058} \mathrm{G}$ in the $23 \mathrm{~S}$ rRNA alleles for tylosin/tilmicosin and of $\mathrm{A} 965 \mathrm{~T}$ and $\mathrm{A} 967 \mathrm{~T}$ in the $16 \mathrm{~S}$ rRNA alleles for oxytetracycline. The genotypes of ST3 or ST1 isolates ( $n=9$ and 25, respectively) in the process of becoming resistant were more varied. In recent years, the convergence of both ST2 and ST3 isolates toward the same resistance genotype suggests that the corresponding mutations have been selected for providing an appropriate balance between fitness cost and resistance.

Keywords: drug resistance, mycoplasma, macrolides, tetracyclines

Introduction

T

t has recently become evident that mycoplasmas are no exception to the dramatic increase in the overall resistance of bacteria to antimicrobials worldwide. These particular bacteria, characterized by a small genome and small cell size, are important pathogens in both human and veterinary medicine. Due to their lack of a cell wall, they are naturally resistant to b-lactams and glycopeptides. Furthermore, losses in susceptibility to drugs such as tetracyclines, fluoroquinolones, and macrolides have been extensively reported during the past decade in many clinically relevant species. A data compilation, on phenotypic resistance and the underlying molecular mechanisms in mycoplasmas of both human and animal origin, is provided in the recent review by Waites et al.1

Marked differences in this recent evolution toward resistance are apparent, depending on the mycoplasmal species, the animal host, the antimicrobial exposure of the concerned population, and the country of origin of the isolates. For instance, the acquired resistance to tetracyclines in Mycoplasma hominis, a genital human mycoplasma occasionally involved in infections, has long been known and associated with the tet(M) determinant carried on the conjugative transposon Tn916, but has not yet been reported in Mycoplasma pneumoniae, the causative agent of community-acquired respiratory tract infections in children and young adults.2,3 Acquired resistance to macrolides in $M$. pneumoniae, is still regarded as a rare and geographically localized phenomenon, like in Asia,1,3 whereas in animals, the rapid emergence of resistant isolates has been reported in different species with a highly critical situation in the bovine mycoplasma, $M y$ coplasma bovis.1,2 Indeed, as summarized in a recent article by Heuvelink et al.,4 the minimum inhibitory concentrations (MICs) of macrolides for almost all the $M$. bovis isolates collected after the year 2000, worldwide, were $>64 \mathrm{mg} / \mathrm{ml}$.

Among the many species of mycoplasmas of veterinary importance, $M$. bovis is considered an emerging threat to 
livestock production in industrialized countries.5 It is a pathogen of cattle that is mainly responsible not only for bronchopneumonia in young animals but also for other clinical manifestations, for example, mastitis, otitis, and arthritis of variedimportancein different countries. 6 In the absence of effective vaccines, 7,8 the control of $M$. bovis mycoplasmosis reliesongoodhusbandrypracticesandefficientantimicrobial treatments. However,in bovine respiratory diseases, frequent co-infections with other microorganisms are associated with diverse levels of infection severity9 and lead to the frequent use of multiple antimicrobials, mainly penicillins, cephalosporins, tetracyclines, macrolides, and quinolones.10 Mycoplasma mastitis and arthritis in cattle are considered poorly responsive to chemotherapy and in the absence of a specific Mycoplasma diagnosis, these clinical signs are often treated with betalactams as a primary strategy.6,11

Tetracyclines and macrolides are classes of antimicrobials that are widely used in veterinary medicine, and especially in ruminants. They are both bacteriostatic drugs that block protein synthesis by (1) preventing linkage of aminoacyltRNA to the acceptor site through binding to the $30 \mathrm{~S}$ ribosomal subunit involving $16 \mathrm{~S}$ rRNA for tetracyclines12,13 or (2)causingprematuredissociationofpeptidyl-tRNAfromthe ribosome by binding to domains II and V of $23 \mathrm{~S}$ ribosomal RNA in the 50S ribosomal subunit for macrolides.14,15 The pharmacokinetic properties of macrolides enable them to concentrate and persist in tissues, especially the lungs, thus justifying the use of a single dose for the semisynthetic tilmicosin and tulathromycin.14 Hence, macrolide-based drugs often have a specific indication for M. bovis, a common agent of bovine respiratory disease, whereas tetracycline-based chemotherapy has a broader target such as septicemia, respiratory, digestive, or reproductive disorders due to susceptible bacteria in cattle. In general, tetracylines are widely usedinfoodanimalsasfirst-linedrugs, fortheirlowcost,ease of use, and wide range of action.12 The index of veterinary drugs authorized in France can be consulted at www.ircp .anmv.anses.fr.

As expected from the intensive use of antimicrobials, reportsofan invitro decreaseinthesusceptibilityof M.bovis to several antimicrobial classes have started to accumulate worldwide. 4 In France, all clinical isolates collected between 2009 and 2012 were shown to have gained resistance to all antimicrobials except fluoroquinolones when compared with isolates dating back to 1978-1979.16 This emergence of isolates with reduced susceptibility was further linked to the spread of a homogeneous population of strains with poor genetic diversity, belonging to a major subtype.17 The only antimicrobial family spared was the fluoroquinolones, although a first clinical isolate with increased MIC was identified in 2013.18 In the absence of susceptibility breakpoints for $M$. bovis, the clinical interpretation of in vitro results is often not possible. However, breakpoints available for other bacteria that are responsible for respiratory diseases in cattle canbeusedforapproximation. ${ }_{19}$ Forinstance, themeanMICs for macrolides and tetracyclines in all recent isolates from France were greater than the Clinical and Laboratory Standards Institute (CLSI) breakpoints proposed for Pasteurellaceae and, hence, could be considered resistant.16 The mechanisms behind this loss of susceptibility have not been examined for French isolates, and only a few studies have been conducted so far on the resistance mechanisms to macrolides and tetracyclines in M. bovis.20-22 These studies essentially reported sequence alterations in $23 \mathrm{~S}$ (domains II and V) and $16 \mathrm{~S}$ rRNAs, respectively. More precisely the A 2058 G substitution (Escherichia coli numbering) in domain $\mathrm{V}$ of $23 \mathrm{~S}$ rRNA, already described in M. pneumoniae, 3,21 is reported for macrolides. Resistance to tetracyclines was associated with two (A965 and A967) or three positions (A965, A967, and $\mathrm{G}_{1058)}$ of mutations in the two 16S rRNA copies, as already shown for other human mycoplasmas.20,23 Various other mutations in ribosomal proteins L4 and L22 were also identifiedthathadbeenrecentlyassociatedwiththedecreased binding of macrolides to ribosomal subunits. 24

The present study was conducted to examine the evolution overtimeoftheemergenceof M.bovis isolateswithincreased MICs for two 16-membered macrolides dedicated to veterinary use (tylosin and tilmicosin) and tetracyclines (oxytetracycline). Sequence analyses were conducted to link alterationsinthetargetregionsof $16 \mathrm{SrRNA}$ ( $r r$ alleles), $23 \mathrm{~S}$ rRNA( $r r l$ alleles), andL4andL22proteins(encodedby $r p l D$ and $r p l V$, respectively) with resistance phenotypes. The objective was to understand the time lapse and molecular mechanisms underlying the previously observed shift from a highly susceptible population in 1978-1979 to a highly resistant one in 2012.16 Isolates were grouped according to clinical signs (respiratory disease vs. other origins) to determine whether differences in therapeutic practices and antimicrobial uses could influence the susceptibility profiles. All isolates were further subtyped by a single-locus sequence typing approach 17 to establish their epidemiological relationships and, hence, the main evolutionary trends within the M. bovis population in France.

Materials and Methods

Mycoplasma isolates, growth, identification, and subtyping

Seventy-four $M$. bovis clinical isolates collected from 31 "de'partements" of France were included in this study. They originated from a collection maintained in Anses Lyon and mostly derived from the French national surveillance network for mycoplasmoses of ruminants (VIGIMYC).25 They were isolated from young cattle with respiratory diseases (40 isolates, Table 1) or from cattle with nonrespiratory clinical signs such as mastitis $(n=15)$, otitis $(n=4)$, arthritis $(n=11)$, or unknown $(n=4)$ (Supplementary Table S1; Supplementary Data are available online at www.liebertpub .com/mdr). The type strain PG45т (isolated in the United States in 1962 from a mastitis case) and two isolates from Switzerland (collected in 2010 from cases of respiratory disease26) were added as external controls. All isolates were grown in pleuropneumonia-like organism (PPLO) broth or agar, modified as previously described, 27 at $37^{\circ} \mathrm{C}$ in $5 \% \mathrm{CO}_{2}$. To standardize the mycoplasma inocula used for MIC tests (see hereafter), the number of colony forming unit (CFU) per $\mathrm{ml}$ was determined by plating $2 \mathrm{ml}$ of serial 10 -fold broth dilutions onto agar plates. After incubation for 3 days, the colonies for dilutions resulting in countable, nonconfluent spotswerecountedbyusingastereomicroscopeandthemean finalcellconcentrationwasdeterminedbyusingtwodilutions that resulted in cell count within a range comprising between 10 and 300. Isolates were identified by membrane filtration 


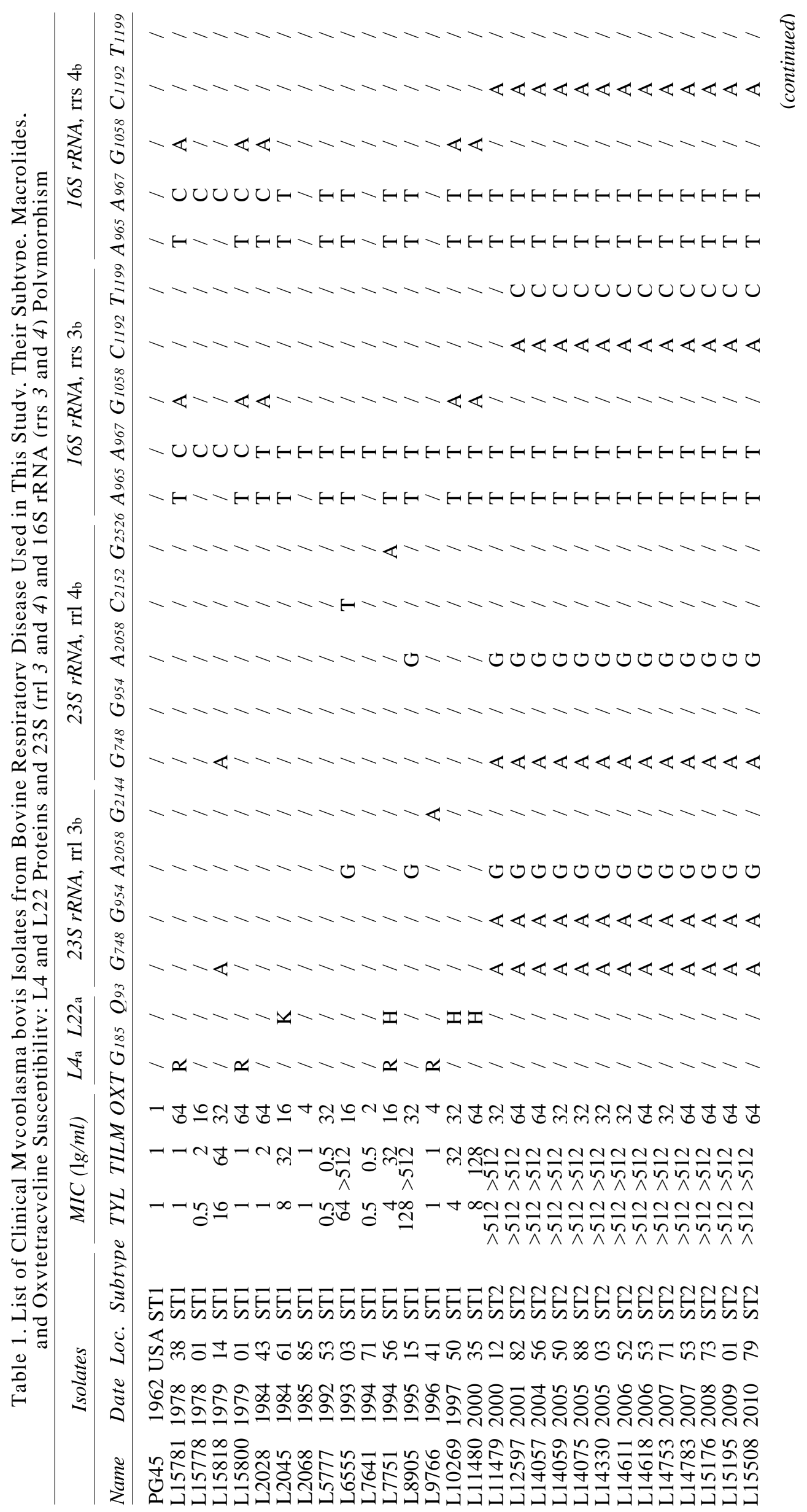




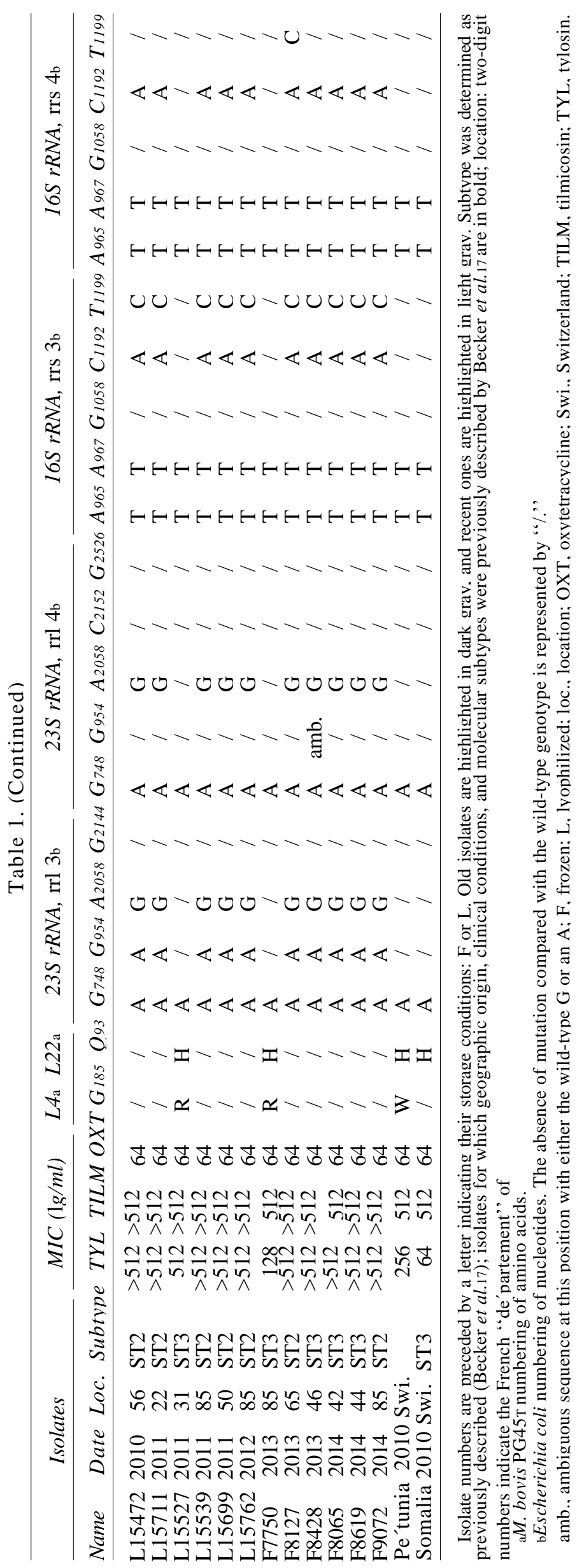


dot-immunobinding tests 28 and by a species-specific PCR assay targeting the polC gene.29 They were further subtyped by a single-locus sequence analysis (a 486bp region of the polC gene) that had been shown to provide relevant typing results.17 The primers and PCR conditions used for these assays are listed in Supplementary Table S2.

Minimum inhibitory concentration assays

For respiratory isolates, the MICs of tylosin, tilmicosin, and oxytetracycline, all purchased from Sigma-Aldrich France, were determined by using the agar dilution method on modified PPLO agar, as previously described.16,18 In brief, $1 \mathrm{ml}$ of each strain diluted to $10_{4}-10_{5} \mathrm{CFU} / \mathrm{ml}$ was spotted onto agar plates containing serial twofold dilutions of each antimicrobial (from 0.25 to $512 \mathrm{mg} / \mathrm{ml}$ for macrolides; from 0.25 to $64 \mathrm{mg} / \mathrm{ml}$ for oxytetracycline) and grown at $37^{\circ} \mathrm{C}$ in $5 \% \mathrm{CO}_{2}$ for 5 days. The MIC was defined as the lowest antimicrobial concentration causing $100 \%$ inhibition of growth after the incubation period.30 MIC assays were performed at least twice and three times in case of different MIC values for each isolate, and the MIC values given in the results section are the mode of the different values. For isolates collected from nonrespiratory cases, only one to three different concentrations were used per antimicrobial. These concentrations corresponded to the epidemiological cut-off determined on the basis of the MIC distributions for each antimicrobial and our set of respiratory isolates used as reference (Fig. 1). They were $8 \mathrm{mg} / \mathrm{ml}$ for oxytetracycline and $16 \mathrm{mg} / \mathrm{mlfortilmicosinandtylosin}$. Theywereidenticaltothe CLSI breakpoints defined for Pasteurellaceae for tilmicosin and tetracyclines 31 and to the general MIC breakpoint proposed for tetracyclines in the review by Hannan.30 Two supplementaryconcentrationsof 1 and $256 \mathrm{~m} \mathrm{~g} / \mathrm{ml}$ wereadded to better discriminate isolates that were, respectively, highly susceptible or resistant to macrolides.

Polymerase Chain Reaction amplification and sequence analysis of rrs3, rrs4, rrl3, rrl4, rplD, and rplV genes

Genomic DNA was extracted from $2 \mathrm{ml}$ logarithmicphase broth cultures of $M$. bovis by using a commercial kit (Blood and tissue kit; Qiagen, France). Because of the duplication of the $16 \mathrm{~S}$ rRNA/23S rRNA locus in M. bovis PG45T, a nested PCR approach was developed to specifically amplifyeachindividualcopy(Fig.2).Threelong-rangePCRs were first run on genomic DNA. These included two previously described assays using primers MB-282-F/MB-rrl-3R andMB-rrl-II-F/MB-287-R 21 togeneratePCRproducts1 and 2andathirdhome-designedPCRthattargetsthesecondcopy of 16S rRNA and part of the first 23S rRNA copy, using primers23S-V1-FandU8(PCR3inFig.2).PCRproducts 13 were then used as template, after 1/100 dilution, to run the severalnestedPCRslistedinSupplementaryTableS2.Genes encoding ribosomal proteins, $r p l D$ and $r p l V$ for proteins L4 andL22, respectively, weretentativelyamplifiedbyusingthe protocol proposed by Lerner et al.21 However, in our experimental set up using the GoTaq polymerase from Promega (France), we failed toobtain a single amplicon using MB-L4F and MB-L4-R primers. Hence, two new primers (L4F and L4R) were designed. Details of the individual PCR run conditions are given in Supplementary Table S2. PCR products corresponding toindividual copiesofeachrRNAand domain were sequenced by using an external facility at Beckman CoulterGenomics(Genewiz, UK).Thelengthandnucleotide upper and lower boundaries of the analyzed sequences (positioned in Fig. 2) are given in Supplementary Table S2. Sequence editing, consensus, and alignment construction were performed by using Seaview software (http://doua.prabi.fr/ software/seaview)andGeneious version6.1.8 (www.geneious. com). For convenience, the nucleotide numbering refers to that of E. coli and the amino acid numbering for the L4 and L22 proteins to that of M. bovis PG45т. For the second set of isolates, only 26 out of 34 isolates, selected as representing the different subtypes and MIC levels, were analyzed for their resistance genotype.

\section{Results}

Distribution of minimum inhibitory concentrations for isolates from respiratory diseases

A set of 40 clinical isolates of $M$. bovis, collected between 1978 and 2014, exclusively from respiratory diseases, was assembled from our collection (Table 1). These isolates were epidemiologicallyunrelated, astheyoriginatedfromdifferent outbreaks, different regions, and different years. Isolates collectedbetween1978and1997( $n=14)$ arehereafternamed "old," whereas those collected from 2000 to $2014(n=26)$ are named "recent." Two isolates collected in 2010 from neighboring Switzerland were added as external controls.26

The susceptibility of these 40 isolates to the 16-membered macrolides, tylosin and tilmicosin, and to oxytetracycline was determined by MIC assays using the agar dilution method according to the recommendations of the CLSI.31 The type strain, PG45T with known MICs was used as a quality control in each assay. It was confirmed to be mainly susceptible with MIC values of $1 \mathrm{mg} / \mathrm{ml}$ for tylosin, tilmicosin, and oxytetracycline (black arrows in Fig. 1). The distribution of the MIC values of tylosin, tilmicosin, and oxytetracycline for French isolates is shown in Fig. 1, where old isolates are represented by gray bars and recent isolates are represented by white bars. This representation clearly illustrates the shift over time toward less susceptible isolates, although the first isolates with increased MICs for both oxytetracyline and macrolides were isolated as early as 1979. For all recent isolates from France, the MICs of tilmicosin and oxytetracycline were greater than the CLSI breakpoints proposed for Pasteurellaceae and, hence, could be considered resistant (Fig. 1). The MIC distribution of tylosin, for which no interpretation breakpoints are available, highlighted four different groups of isolates. Old ones were scattered in three populations, one between 0.5 and $1 \mathrm{mg} / \mathrm{ml}$ for very sensitive isolates (8/14), a second between 4 and $16 \mathrm{mg} / \mathrm{ml}$ for intermediate isolates (4/14), and a third between 64 and $128 \mathrm{mg} / \mathrm{ml}(2 / 14)$. In contrast, all recent isolates, except one (namely, isolate no. L11780, collected in 2000 with an MIC of $8 \mathrm{mg} / \mathrm{ml}$; highlighted by a star in Fig. 1), had MICs $\$ 128 \mathrm{mg} / \mathrm{ml}$, with most (24/26) being highly resistant with MICs $₫ 512 \mathrm{mg} / \mathrm{ml}$. The overall distribution of tilmicosin MICs was similar except that no intermediate isolates, according to the MIC breakpoints proposed by the CLSI, were observed and two old isolates, collected in 1993 and1996, werealreadyhighlyresistantwithanMIC > $512 \mathrm{mg} /$ $\mathrm{ml}$. For recent isolates, all MICs of tilmicosin were $\$ 512 \mathrm{mg} /$ 
FIG. 1. Comparison of MICs distributions of three antimicrobials for 40 Mycoplasma bovis isolates collected from bovine respiratory diseases in France between 1978 and 2014. Old isolates (1978-1997, $n=14)$ and recent ones (2000-2014, $n=26$ ) are represented by gray and white bars, respectively. The number of isolates per MIC data is indicated in the bars. $x$-axis, MICs in $\mathrm{mg} / \mathrm{ml} ; y$ axis, \% of isolates. Black and open arrows indicate MICs for PG45T and Swiss strains, respectively. Double arrows below the $x$-axis indicate the epidemiological cut-off for each antimicrobial. The asterisk indicates the position of the isolate collected in year 2000 but belonging to subtype 1 (no. L11480). The S, I, and R sections correspond to isolates categorized as Susceptible, Intermediate, or Resistant by using the CLSI breakpoints (when available) for other bacteria that are responsible for respiratory diseases in cattle. MICs, minimal inhibitory concentrations.
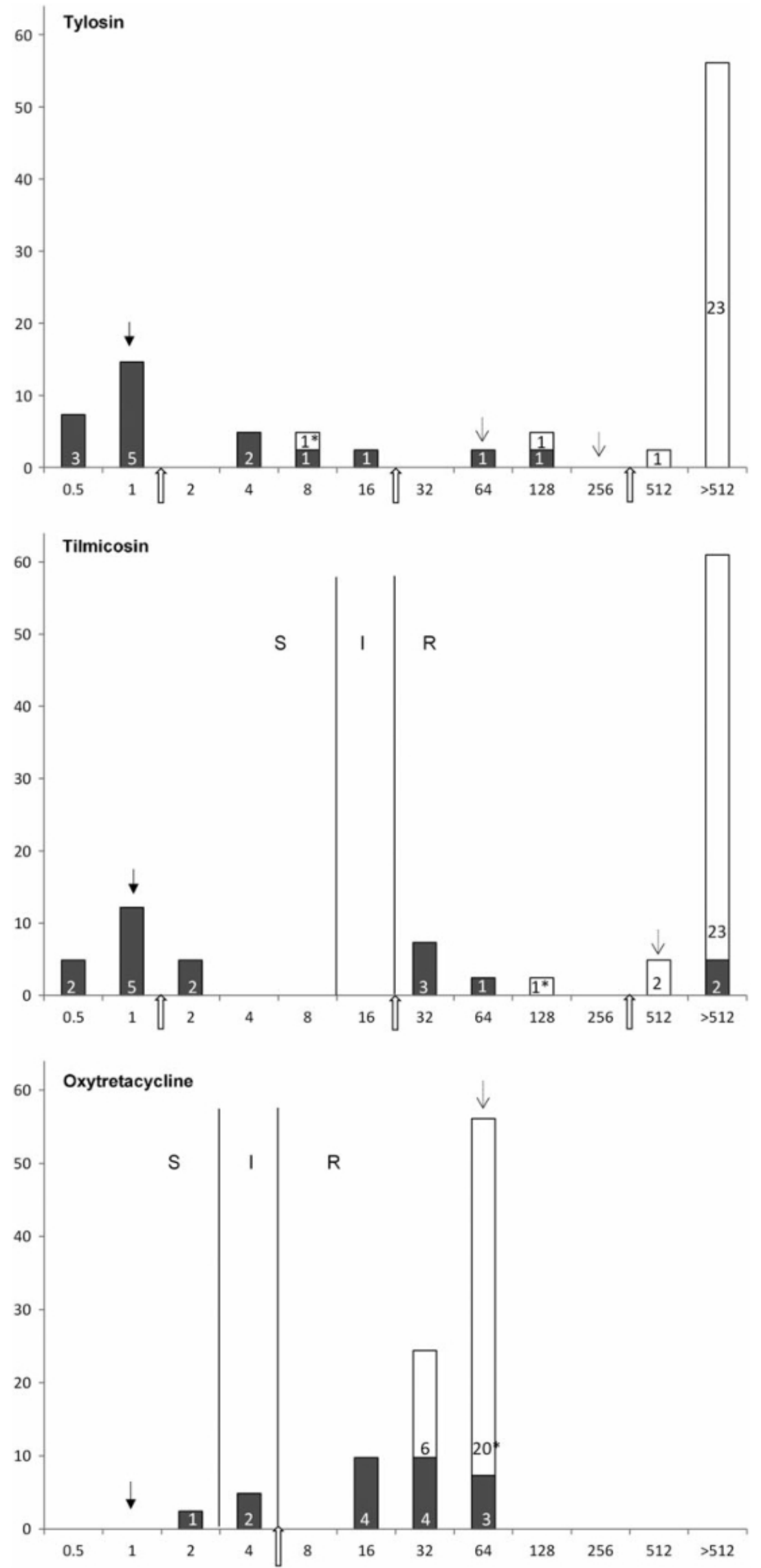

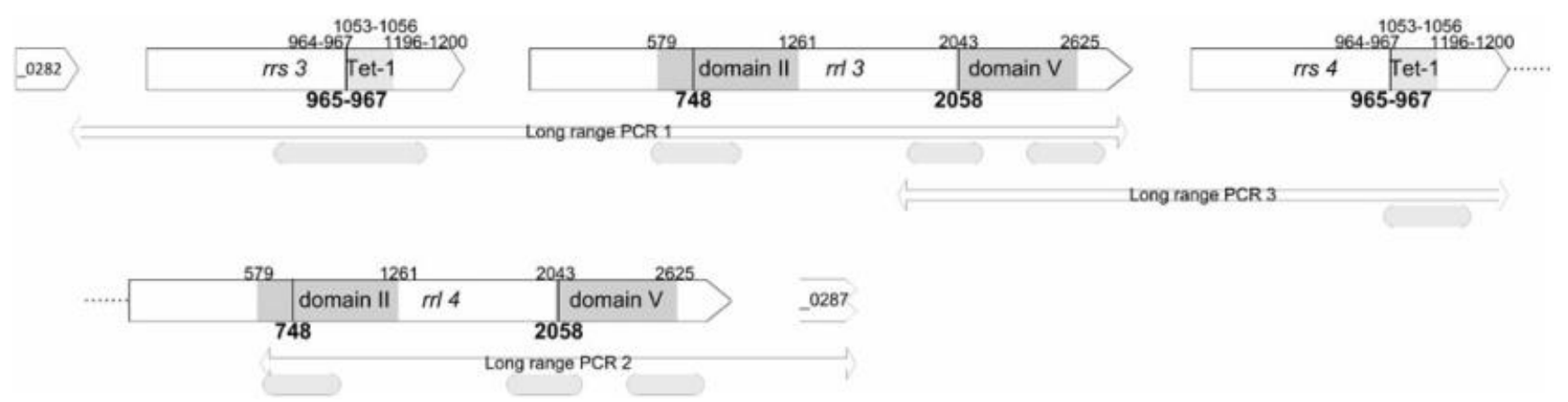

FIG. 2. Graphical representation of the $r r s$ and $r r l$ genes regions of $M$. bovis. Genes are represented by arrows and named after their locus tag in PG45т. Gray squares highlight the binding domains of both tetracyclines (rrs) and macrolides $(r r l)$, and mutation hot spots are indicated by black lines. The long-range PCR assays used in the study are positioned by double arrows. Curved edge boxes indicate the sequences that were analyzed (for exact position, see Supplementary Table S2).

$\mathrm{ml}$, except the isolate collected in 2000 that had an MIC of $128 \mathrm{mg} / \mathrm{ml}$. In contrast, for oxytetracycline, the distinction between old and recent isolates was less evident, as most of the old isolates (11/14) should be classified as resistant by using the general MIC breakpoints for cattle respiratory disease bacteria from CLSI $(\$ 8 \mathrm{mg} / \mathrm{ml})$. Only three old isolates should be considered susceptible (L7641, MIC $£ 2 \mathrm{mg} / \mathrm{ml}$ ) or intermediate (L2068 and L9766, MIC $=4 \mathrm{mg} / \mathrm{ml}$ ). All recent isolates were also resistant, with MIC values $\$ 32 \mathrm{mg} / \mathrm{ml}$. The two Swiss isolates fell into the category of recent French isolates, with MIC values of $64-256 \mathrm{mg} / \mathrm{ml}$ for tylosin, $512 \mathrm{mg} / \mathrm{ml}$ for tilmicosin, and $64 \mathrm{mg} / \mathrm{ml}$ for oxytetracycline (open arrows in Fig. 1).

\section{Ribosomal targets polymorphism}

To elucidate the molecular basis underlying the different susceptibilities of old and recent isolates, the sequences of domains II and V of the 23S rRNA, ribosomal proteins L4 and L22, targeted by macrolides, and of the 16S rRNA, targeted by tetracyclines, were analyzed. In each case, these sequences cover the main binding sites and alreadydescribed hot spots for mutations conferring resistance (Fig. 2). Either already described PCR assays or newly designed ones were used. The design of the new primer sets was based on the sequence of the type strain PG45T, which harbors two copies of the $r r l$ gene encoding 23S rRNA and the rrs gene encoding 16S rRNA. The PCR results showed that all our clinical isolates also had two $r r s$ and $r r l$ alleles. Several nucleotide substitutions were observed in all contemporary isolates and in some old ones (Table 1). For the 23S rRNA, two positions were notably altered in both $\mathrm{rrl}$ alleles in a large majority of the recent isolates (23/26), namely $\mathrm{G}_{748} \mathrm{~A}$ in domain II and $\mathrm{A} 2058 \mathrm{G}$ in domain V. These positions have already been described as mutation hot spots in $M$. bovis and other mycoplasma species.1,21,22,32 Another mutation, G954A was observed in recent isolates but only in one copy of 23S rRNA (namely $r r l 3$ ) but had never been reported earlier as a hot spot.1,32 Interestingly, only a few old isolates (3/14) showed the $\mathrm{G}_{748} \mathrm{~A}$ or $\mathrm{A} 2058 \mathrm{G}$ alterations and none had accumulated both point mutations. A few amino acid alterations were observed in the L4 and L22 proteins, namely $\mathrm{G}_{185} \mathrm{R}$ and $\mathrm{Q}_{93} \mathrm{H} / \mathrm{K}$, and were cumulated only in two recent isolates and in one old isolate. Regarding the two 16S rRNA copies (rrs 3 and rrs 4), several nucleotide polymorphisms were observed in five different positions (Table 1). However, they were recurrent only at positions 965 and 967 in both the old (9/14) and recent isolates (26/26). These two positions had previously been described as hot spots for mutation.20 Another repeated mutation point, $\mathrm{G}_{1058 \mathrm{~A}}$, was observed in old isolates (4/14) and in only one recent isolate collected in 2000 .

Relationship between gene alterations in ribosomal targets, minimum inhibitory concentration values, and isolate subtypes

The different subtypes of the isolates were also investigated to determine the potential link between resistant phenotypes, genotypes, andthemolecularsubtypes. Theseisolate subtypes were either already available from a previous study or determined here by using the same approach, that is, sequence analysis of their polC locus.17 The resulting overall profiles (including subtype, level of susceptibility, and resistance genotype) are compared in Fig. 3. As expected from previous results, 17,18 all strains isolated before 1999 were homogeneously grouped in subtype $1(\mathrm{ST} 1, n=14)$ with the type strain PG45T, whereas almost all strains isolated after $2000(19 / 26)$ belonged to subtype 2 (ST2), which diverged from ST1 by a single nucleotide polymorphism (SNP) in the 486bp polC locus. Twostrains, isolatedin2000, haddifferent profiles, one being ST1 and the other ST2, which confirmed previous results that suggested a change in the overall $M$. bovis population in France around 2000.17 Five of the recent isolates (>2000), and the two Swiss strains (isolated in 2010), belonged to ST3, an uncommon subtype characterized by 16 SNPs. 17

The resistance genotypes and MIC values of the ST2 isolates appeared to be more homogeneous than those of the ST1 isolates (Fig. 3). The ST1 isolates were collected in years when resistance to antimicrobials targeting ribosomal RNAs was just emerging and, hence, showed more genetic diversity. At the time of the switch to the ST2-lineage (around year 2000), all isolates had become highly resistant (MICs $>256 \mathrm{mg} / \mathrm{ml}$ for macrolides and $\$ 32 \mathrm{mg} / \mathrm{ml}$ for oxytetracycline) and shared 10-12 common single-nucleotide substitutions in the 23S rRNA and 16S rRNA alleles. These substitutions include hot spot mutations (dark-gray squares), 


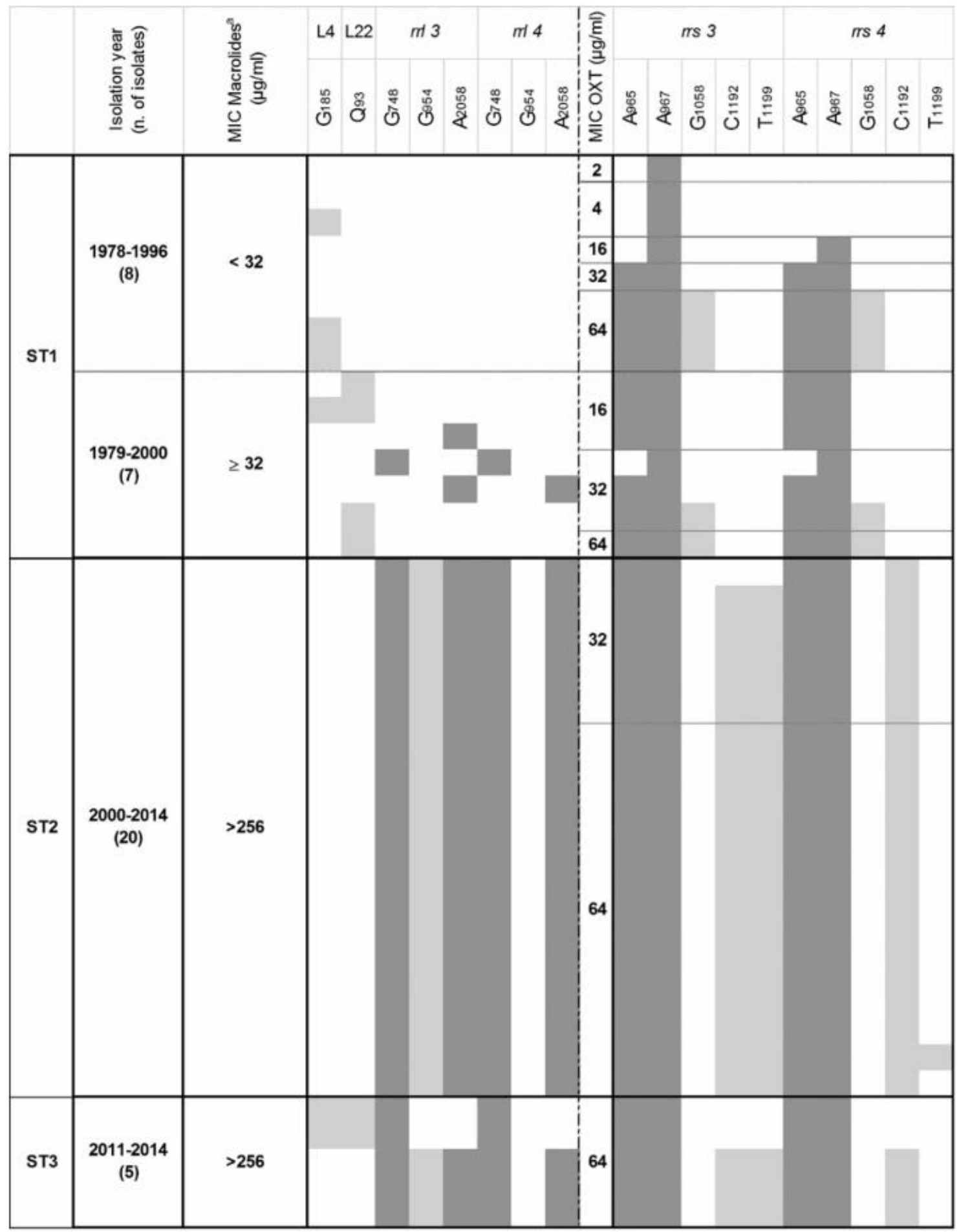

FIG. 3. Relationship between subtypes, sampling year, MIC values, and mutations in target genes of isolates from bovine respiratory diseases. Individual isolates belonging to the different subtypes are each represented by a line. The reference sequence is that of M. bovis PG45 and any acquired mutation is indicated by colored squares, either dark gray for hot spots already described in other studies or light-gray for genetic alterations that had not previously been associated with higher MICs. Localization of the mutations is indicated according to Escherichia coli numbering in the rrs and $r r l$ genes, and according to the M. bovis amino acid sequence for L4 and L22. aThe MIC value indicated refers to the maximum value attained for tylosin and/or tilmicosin. 
previously described in other mycoplasma species $23,33,34$ and in $M$. bovis isolated in other countries.20-22 Other nucleotide substitutions (light-gray squares) were also observed in the target genes but outside, although close to, the main target domains. They had never earlier been correlated with resistance 1,35 but are mentioned in Fig. 3 when they occurred in more than three isolates (included those from nonrespiratory disease). In summary, our results clearly illustrate the shift from a susceptible population with few mutations (ST1-lineage) to a resistant population (ST2lineage) with cumulated mutations in both alleles of $16 \mathrm{~S}$ rRNA and 23S rRNA. Furthermore, we confirmed that double mutations in position $965 / 967$ of both $r r s$ alleles were associated with high MIC values for oxytetracycline (>32 mg/ml) and that double mutations at positions 748/ 2,058 in both $r r l$ alleles were associated with high resistance to 16 -membered macrolides (>512 $\mathrm{mg} / \mathrm{ml})$. Interestingly, the resistance genotypes of isolates belonging to the ST3lineage, although few, were more varied than those of ST2 with, notably, two isolates showing amino acid substitutions in L4 and L 22 but no nucleotide substitution in position 2,058 of the $23 \mathrm{~S}$ rRNA alleles. The genotypes of the two control isolates from Switzerland had also not cumulated alterations in their $r r l$ alleles but displayed mutations in L4 and L22 associated with high MICs (Table 1). This suggests that isolates belonging to the ST3-lineage acquired resistance and mutations over a different timeframe to that of the ST2 isolates.

Comparison with isolates collected

from nonrespiratory diseases

For comparison, $34 \mathrm{M}$. bovis isolates collected from nonrespiratory clinical cases between 1982 and 2015 were also analyzed by using a similar approach. These isolates originated from mastitis, otitis, arthritis, and a few other nondocumented diseases (Supplementary Table S1). They were also classified as old or recent, the recent ones having been collected after 2000. MICs were estimated by using the same one or three concentrations used to delineate the different M. bovis populations from respiratory disease cases (Fig. 1), namely $8 \mathrm{mg} / \mathrm{ml}$ for oxytetracycline and 1, 16, and $256 \mathrm{mg} / \mathrm{ml}$ for macrolides. Interestingly, for oxytetracycline and tilmicosin, the $\$ 8$ and $\$ 32$ (equivalent to $>16$ ) $\mathrm{mg} / \mathrm{ml}$ breakpoints, respectively, correspond to those defined by the CLSI for categorization of resistant bacteria involved in bovine respiratory diseases.31 All recent isolates could be considered resistant to macrolides, whereas old ones $(<2000)$ were susceptible $(7 / 11)$, intermediate $(1 / 11)$, or resistant (3/11) to tilmicosin. This distribution is comparable to that of isolates collected from respiratory diseases. On the other hand, for all isolates, whatever their isolation dates, the MIC for oxytetracycline was $\$ 8 \mathrm{mg} / \mathrm{ml}$ except for two old isolates. The antimicrobial target genes were then sequenced to detect mutations in a subset of 26 isolates representing the different subtypes and MIC levels. Sequence analysis revealed the same substitution points as in isolates from respiratory diseases.Thedistributionsofgenealterations, MICvalues,year, and isolate subtypes are shown in Fig. 4. Because no differences were evidenced between isolates as a function of their clinical history (e.g., mastitis vs. otitis), this distinction was not introduced into the table. Three distinct groups emerged from this classification: (1) ST1-isolates with minor alterationsandlowerMICs(althoughtwowerealreadyresistantto tilmicosin and nine to oxytetracycline), (2) ST2-isolates homogeneously harboring multiple hot-spot mutations and with highMICs, and(3)ST3-isolates, alsowithhighMICsbutthat had more polymorphic $r p l D, r p l V, r r s$, and $r r l$ genes sequences. This general pattern is very similar to that described for isolates from respiratory diseases in Fig. 3.

\section{Discussion}

Although studies to correlate antimicrobial uses in foodproducing animals with the emergence of drug resistance in animal bacteria started long ago, they were hampered by the difficulty of gathering accurate information about antimicrobial consumption of animals.11,36 In contrast, monitoring the evolution of in vitro susceptibility and genetic evidence of resistance over time provides a simple approach for estimating the impact of antimicrobials uses on the evolution of a bacterial population in the long term. We knew from an earlier study that, in France, the MICs of M. bovis isolates for several antimicrobials had increased 16 but the rapidity of this evolution and its genetic determinants had not been determined for the antimicrobials targeting ribosomal RNAs.

First, our results did not evidence any difference between isolates from respiratory or other bovine diseases in terms of genotypic and phenotypic resistance patterns to tetracyclines and macrolides. In other words, the fact that macrolides are mainly used to treat respiratory diseases, 11 unlike the broadspectrum tetracyclines used in a range of therapeutic areas, hadnotledtoamorerapidormarkedresistancetomacrolides in respiratory isolates. The same conclusion was reached in a recent study of the MICs of $M$. bovis isolates from mastitis that were compared with earlier data obtained on respiratory isolates.37 This would argue in favor of an efficient circulation and transmission of resistant isolates between different organs and animals. In contrast, our data illustrate the potential impact of the marketing authorization date, with most old isolates already being resistant to tetracyclines, released as far back as the early 1950s, but not to macrolides.

Applying the CLSI breakpoints, 75\% (20/25) of the $M$. bovis isolates collected before 2000 were already resistant to oxytetracycline and only one isolate was in the Susceptible category (L7641). The four intermediate isolates $(2<\mathrm{MIC}$ $<8 \mathrm{mg} / \mathrm{ml}$ ) harbored a single A967 T mutation in one rrs allele only. This 967 position is located within the primary binding pocket for tetracyclines, which is formed by an irregular minor groove of helix H34 (RNA residues 1,196-1,200:1, 053-1,056) in combination with residues 964-967 from the helix H31 stem-loop.13 It is likely that the nonmutated rrs copy covers the function of the mutant copy,20 explaining why the impact of a single allele mutation on MIC values is minor. All other isolates with MIC $>8 \mathrm{mg} / \mathrm{ml} \mathrm{had} \mathrm{accumu-}$ lated mutations in both positions 965 and 967, and systematically in both alleles for isolates collected after 2000. This suggests that homozygote mutations in positions 965 and 967 of the rrs genes are necessary and sufficient to increase the MICs of oxytetracycline and to categorize such isolates as resistant. Other observed mutations may modify the accessibility of the tetracycline binding pocket but without further modifying the MICs. For instance, the 1,058 position, located outside but next to the groove of helix H34, 


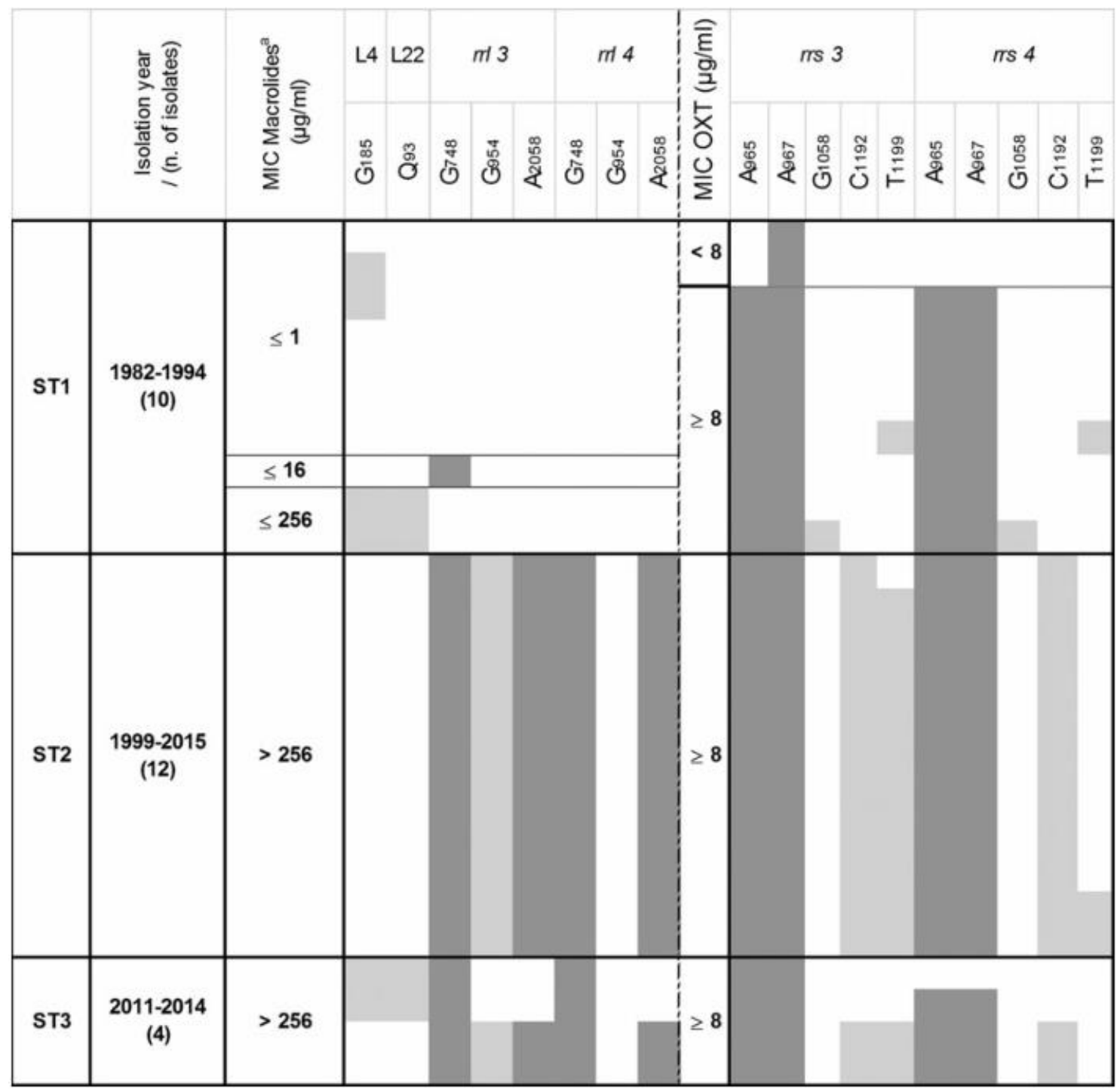

FIG. 4. Relationship between subtypes, sampling year, MIC values, and mutations in target genes of isolates from nonrespiratory diseases. The representation is the same as in Fig. 3. aThe MIC value indicated refers to the maximum value reached for tylosin and/or tilmicosin, $£ 16$ is indicated for strains with $1<\mathrm{MIC} £ 16 \mathrm{mg} / \mathrm{ml}$, and $£ 256$ is indicated for strains with $16<$ MIC $£ 256 \mathrm{mg} / \mathrm{ml}$.

was suggested to potentially impact the conformation of the tetracycline binding pocket and was previously reported to be associated with increased MICs.20 The $\mathrm{G}_{1058} \mathrm{~A}$ mutation was infrequently observed in our sets of isolates (in only six isolates, mainly old ones with the exception of isolate L11480) and was not related to higher MIC values. The same observation was made for the $\mathrm{C}_{1192 \mathrm{~A}}$ alteration present in all recent strains (except six, namely L15527, L11480, L11479, L15517, L15516, and F7750) in both alleles but absent in old strains (except L10971). The presence/absence pattern of the $\mathrm{T}_{1199} \mathrm{C}$ mutation (only one allele) located within H34 is very similar. The 1,192 and 1,199 alterations were also observed in the study by Amram et al. and were proposed to potentially influence the binding of tetracyclines.20 However, our opinion is that they did not influence MIC levels, as they were absent in the two Swiss isolates (that reached MICs of $64 \mathrm{mg} / \mathrm{ml}$ ) but present in isolates of the Amram study where the observed maximum MIC was
$16 \mathrm{mg} / \mathrm{ml}$. Other secondary binding sites for tetracyclines located within the body of the $16 \mathrm{~S}$ subunit have been suggested 13 but were either not explored here (edge of helix $\mathrm{H} 11$, residues 242-245) or had no mutations (at least in one of the two alleles) when compared with the PG45T control isolate (major groove of helix H27, residues 891-894:908911). In summary, the A965 T and A967 T mutations in both rrs 3 and rrs 4 were identified as the main cause of resistance to oxytetracyline in our set of $M$. bovis isolates.

Although macrolides are mycoplasmastatic and, like tetracyclines, work by inhibiting protein synthesis, they differ in their pharmacodynamic properties, including their binding site. They bind within the tunnel of the 50S ribosomal subunit and interact with several key $23 \mathrm{~S}$ rRNA nucleotides, the most common one being A2058 (domain V), with an additional interaction of tylosin-based drugs with and around nucleotide $\mathrm{G}_{748}$ (domain II), and with the surface of proteins L4 and L22.15,38 Tylosin was put on the market 
as early as 1980 , whereas its semisynthetic derivative tilmicosin was introduced later, in 1994. Interestingly, the susceptibility of isolates to these two molecules has evolved differently over time. Taking the $\$ 32 \mathrm{mg} / \mathrm{ml} \mathrm{CLSI} \mathrm{break-}$ point for tilmicosin as reference, the shift to resistance has been faster for tilmicosin than for tylosin with no development of an intermediate population and already highly resistant isolates among those collected before 2000 (9/25 for tilmicosin and 3/25 for tylosin). Both chemicals share the same ribosomal binding site and although their way of occupying is subtly different, 15,39 resistance to tylosin could have co-selected resistance to tilmicosin. Most recent isolates have high MIC values $(\$ 128 \mathrm{mg} / \mathrm{ml})$ and have accumulated both $\mathrm{G}_{748}$ and $\mathrm{A}_{2058}$ mutations in both $\mathrm{rrl}$ alleles. This result essentially confirms previous observations that a combination of mutations in these hot spots of the two domains is necessary and sufficient to attain high MICs.21,38 Interestingly, one isolate collected in 1979 (no. L15818), that is, before the two macrolides were actually used, was already resistant/intermediate to tilmicosin and tylosin with MICs of 64 and $16 \mathrm{mg} / \mathrm{ml}$, respectively. This isolate has accumulated the $\mathrm{G} 748 \mathrm{~A}$ point mutation in both alleles of the $23 \mathrm{~S}$ rRNAs (domain II) but has no mutation in domain $\mathrm{V}$ or in proteins L4 and L22. How this mutation was selected has yet to be determined. Furthermore, according to Novotny et al., a single-point mutation at $\mathrm{G}_{748}$ in $E$. coli has no appreciable effect on the MIC values of tylosin and needs to be combined with a mutation at position 2,058 to attain significantly higher MICs.38 If this also holds in mycoplasmas, then we can suspect the contribution of other resistance mechanisms (such as ribosomal methylation and drug efflux) in the evolution of old isolates toward resistance. However, this possibility must be considered with caution, as the L15818 isolate is the only one supporting this hypothesis. Several other old isolates (L10269, L2045, L6129, L6692, and L11480 isolated in 2000 but belonging to ST1) showed increased MIC values but had no mutations at all in their $r r l$ genes. Their L22 protein was mutated at position $93(\mathrm{Q} 93 \mathrm{H} / \mathrm{K})$, which, despite being located far from the macrolides-binding site, could influence its conformation. In contrast, the G $185 \mathrm{R}$ mutation in L4 alone had no effect on the MICs (L1067 and F3213 with MICs $£ 1$ mg/ml), contrary to a previous suggestion.21 Our results further imply that L4 and L22 mutations are associated with high MIC values if and only if combined with $\mathrm{rrl}$ alterations, as suggested for other mycoplasmas 34 and for M. bovis isolates from another country.21 For instance, four ST3 isolates, six if the two isolates from Switzerland are taken into account, had MICs $\$ 64 \mathrm{mg} / \mathrm{ml}$ for tylosin and tilmicosin and harbored the combined alterations of $\mathrm{G}_{185}$ (in L4) and/or Q93 (in L22) and $\mathrm{G}_{748 \mathrm{~A}} \mathrm{~A}$ (both alleles).

The shift in the M. bovis population from the molecular subtype ST1 (old isolates) to the recent, predominant subtype ST2 was associated with a homogeneous increase of MICs and a common resistant genotype (combination of the $\mathrm{G}_{748} \mathrm{~A}$ and $\mathrm{A} 2058 \mathrm{G}$ alterations in the $23 \mathrm{~S}$ rRNA alleles for tylosin and that of $\mathrm{A} 965 \mathrm{~T}$ and $\mathrm{A} 967 \mathrm{~T}$ in the $16 \mathrm{~S}$ rRNA alleles for oxytetracycline), suggesting that the clonal spreading described earlier17 was concomitant with or had just followed the acquisition of resistance. In contrast, the timeframe of evolution to resistance in the ST3 isolates, with more varied genotypes and a wider MIC range, suggests that the emergence of this subtype preceded the acquisition of resistance. In a previous study, ST3 isolates were shown to rapidly and efficiently gain a high level of resistance to fluoroquinolones in vitro.18 This had been hypothesized to result from a greater capacity to cumulate compensatory mutations that counteracted the fitness loss due to antimicrobial-induced mutations. The present data confirm the better capacity of ST3 isolates (vs. ST2) to cumulate sequence alterations (point mutations or indels) in their $r r s$, $r r l, r p l D$, and $r p l V$ genes, both inside and outside the main binding domains (data not shown). We showed here that within a very short period of time (from 2011 to 2014), ST3 isolates have evolved toward the same homogeneous resistance genotype as ST2, for both macrolides and tetracyclines. The hypothesis of a delayed gain in resistance for the ST3-lineage postemergence needs to be further confirmed by including more isolates of ST3 subtypes. Nonetheless, the convergence of both subtypes toward the same resistance genotype suggests that the observed mutations - in brief, A965T and A967T for tetracyclines and $\mathrm{G}_{748 \mathrm{~A}}$ and A $2058 \mathrm{G}$ for tylosin-based drugs-have been selected and, hence, might provide the best balance between the fitness cost of gene alterations and gain in resistance.

\section{Acknowledgments}

The authors thank Agne's Tricot and Ade'lie Colin for their excellent technical assistance, Franc sois Poumarat and all the members of the VIGIMYC network for supplying several M. bovis isolates with documented clinical histories. They are also grateful to Paola Pilo, who kindly provided isolates from Switzerland. D.K.'s PhD research was jointly funded by the French Agency for Food, Environmental and Occupational Health and Safety (Anses) and the Institute of Higher Education in Food Science, Animal Health, Agricultural and Environmental Sciences (VetAgro Sup).

\section{Disclosure Statement}

No competing financial interests exist.

\section{References}

1. Waites, K.B., I. Lysnyansky, and C.M. Be'be'ar. 2014. Emerging antimicrobial resistance in Mycoplasmas of humans and animals. In G.F. Browing and C. Citti (ed.), Mollicutes-molecular biology and pathogenesis. Caister Academic Press, Norfolk, United Kingdom, pp. 289-322.

2. Be'be'ar, C.M., and I. Kempf. 2005. Antimicrobial therapy and antimicrobial resistance. In A. Blanchard and G. Browning (ed.), Mycoplasmas molecular biology pathogenicity and strategies for control. Horizon Bioscience, Wymondham, United Kingdom, pp. 535-568.

3. Pereyre, S., J. Goret, and C. Be'be'ar. 2016. Mycoplasma pneumoniae: current knowledge on macrolide resistance and treatment. Front. Microbiol. 7:974.

4. Heuvelink, A., C. Reugebrink, and J. Mars. 2016. Antimicrobial susceptibility of Mycoplasma bovis isolates from veal calves and dairy cattle in The Netherlands. Vet. Microbiol. 189:1-7.

5. Nicholas, R.A. 2011. Bovine mycoplasmosis: silent and deadly. Vet. Rec. 168:459-462.

6. Maunsell, F.P., A.R. Woolums, D. Francoz, R.F. Rosenbusch, D.L. Step, D.J. Wilson, and E.D. Janzen. 2011. 
Mycoplasma bovis infections in cattle. J. Vet. Intern. Med. 25:772-783.

7. Mulongo, M., T. Prysliak, and J. Perez-Casal. 2013. Vaccination of feedlot cattle with extracts and membrane fractions from two Mycoplasma bovis isolates results in strong humoral immune responses but does not protect against an experimental challenge. Vaccine 31:1406-1412.

8. Soehnlen, M.K., A. Aydin, E.J. Lengerich, B.A. Houser, G.D. Fenton, H.R. Lysczek, C.M. Burns, L.I. Byler, A.L. Hattel, D.R. Wolfgang, and B.M. Jayarao. 2011. Blinded, controlled field trial of two commercially available $M y c o$ plasma bovis bacterin vaccines in veal calves. Vaccine 29: 5347-5354.

9. Burki, S., J. Frey, and P. Pilo. 2015. Virulence, persistence and dissemination of Mycoplasma bovis. Vet. Microbiol. 179: $15-22$.

10. Apley, M.D., and J.F. Coetzee. 2013. Antimicrobial drug use in cattle. In S. Gigue re, J.F. Prescott, and P.M. Dowling (ed.), Antimicrobial therapy in veterinary medicine. 5th ed. John Wiley \& Sons, Inc., Hoboken, pp. 495-518.

11. De Briyne, N., J. Atkinson, L. Pokludova, and S.P. Borriello. 2014. Antibiotics used most commonly to treat animals in Europe. Vet. Rec. 175:325.

12. del Castillo, J.R.E. 2013. Tetracyclines. In S. Gigue`re, J.F. Prescott, and P.M. Dowling (ed.), Antimicrobial therapy in veterinary medicine. 5th ed. John Wiley \& Sons, Inc., Hoboken, pp. 257-268.

13. Brodersen, D.E., W.M. Clemons, Jr., A.P. Carter, R.J. Morgan-Warren, B.T. Wimberly, and V. Ramakrishnan. 2000. The structural basis for the action of the antibiotics tetracycline, pactamycin, and hygromycin B on the $30 \mathrm{~S}$ ribosomal subunit. Cell 103:1143-1154.

14. Gigue`re, S. 2013. Macrolides, Azalides, and Ketolides. In S. Gigue 're, J.F. Prescott, and P.M. Dowling (ed.), Antimicrobial therapy in veterinary medicine. 5th ed. John Wiley \& Sons, Inc., Hoboken, pp. 211-231.

15. Poehlsgaard, J., N.M. Andersen, R. Warrass, and S. Douthwaite. 2012. Visualizing the 16-membered ring macrolides tildipirosin and tilmicosin bound to their ribosomal site. ACS Chem. Biol. 7:1351-1355.

16. Gautier-Bouchardon, A.V., S. Ferre, D. Le Grand, A. Paoli, E. Gay, and F. Poumarat. 2014. Overall decrease in the susceptibility of Mycoplasma bovis to antimicrobials over the past 30 years in France. PLoS One 9:e87672.

17. Becker, C.A., F.M. Thibault, M.A. Arcangioli, and F. Tardy. 2015. Loss of diversity within Mycoplasma bovis isolates collected in France from bovines with respiratory diseases over the last 35 years. Infect. Genet. Evol. 33:118126.

18. Khalil, D., C.A. Becker, and F. Tardy. 2016. Alterations in the Quinolone resistance-determining regions and Fluoroquinolone resistance in clinical isolates and laboratory-derived mutants of Mycoplasma bovis: not all genotypes may be equal. Appl. Environ. Microbiol. 82: 1060-1068.

19. Gerchman, I., S. Levisohn, I. Mikula, and I. Lysnyansky. 2009. In vitro antimicrobial susceptibility of Mycoplasma bovis isolated in Israel from local and imported cattle. Vet. Microbiol. 137:268-275.

20. Amram, E., I. Mikula, C. Schnee, R.D. Ayling, R.A. Nicholas, R.S. Rosales, S. Harrus, and I. Lysnyansky. 2015. $16 \mathrm{~S}$ rRNA gene mutations associated with decreased susceptibility to tetracycline in Mycoplasma bovis. Antimicrob. Agents Chemother. 59:796-802.
21. Lerner, U., E. Amram, R.D. Ayling, I. Mikula, I. Gerchman, S. Harrus, D. Teff, D. Yogev, and I. Lysnyansky. 2014. Acquired resistance to the 16-membered macrolides tylosin and tilmicosin by Mycoplasma bovis. Vet. Microbiol. 168:365-371.

22. Kong, L.C., D. Gao, B.Y. Jia, Z. Wang, Y.H. Gao, Z.H. Pei, S.M. Liu, J.Q. Xin, and H.X. Ma. 2015. Antimicrobial susceptibility and molecular characterization of macrolide resistance of Mycoplasma bovis isolates from multiple provinces in China. J. Vet. Med. Sci. 78:293-296.

23. Degrange, S., H. Renaudin, A. Charron, S. Pereyre, C. Bebear, and C.M. Bebear. 2008. Reduced susceptibility to tetracyclines is associated in vitro with the presence of $16 \mathrm{~S}$ rRNA mutations in Mycoplasma hominis and Mycoplasma pneumoniae. J. Antimicrob. Chemother. 61:1390-1392.

24. Lovmar, M., K. Nilsson, E. Lukk, V. Vimberg, T. Tenson, and M. Ehrenberg. 2009. Erythromycin resistance by L4/ L22 mutations and resistance masking by drug efflux pump deficiency. EMBO J. 28:736-744.

25. Chazel, M., F. Tardy, D. Le Grand, D. Calavas, and F. Poumarat. 2010. Mycoplasmoses of ruminants in France: recent data from the national surveillance network. BMC Vet. Res. 6:32.

26. Aebi, M., M. Bodmer, J. Frey, and P. Pilo. 2012. Herdspecific strains of Mycoplasma bovis in outbreaks of mycoplasmal mastitis and pneumonia. Vet. Microbiol. 157: 363-368.

27. Poumarat, F., D. Longchambon, and J.L. Martel. 1992. Application of dot immunobinding on membrane filtration (MF dot) to the study of relationships within " $M$. mycoides cluster" and within "glucose and arginine-negative cluster' of ruminant mycoplasmas. Vet. Microbiol. 32:375390.

28. Poumarat, F., B. Perrin, and D. Longchambon. 1991. Identification of ruminant mycoplasmas by dot immunobinding on membrane filtration (MF dot). Vet. Microbiol. 29:329-338.

29. Marenda, M.S., E. Sagne, F. Poumarat, and C. Citti. 2005. Suppression subtractive hybridization as a basis to assess Mycoplasma agalactiae and Mycoplasma bovis genomic diversity and species-specific sequences. Microbiology 151: 475-489.

30. Hannan, P.C. 2000. Guidelines and recommendations for antimicrobial minimum inhibitory concentration (MIC) testing against veterinary mycoplasma species. International Research Programme on Comparative Mycoplasmology. Vet. Res. 31:373-395.

31. CLSI. 2013. Performance standards for antimicrobial disk and dilution susceptibility tests for bacteria isolated from animals; approved standard-4th ed. CLSI Document VET01-A4. Clinical and Laboratory Standards Institute, Wayne, PA.

32. Lysnyansky, I., and R.D. Ayling. 2016. Mycoplasma bovis: mechanisms of resistance and trends in antimicrobial susceptibility. Front. Microbiol. 7:595.

33. Matsuoka, M., M. Narita, N. Okazaki, H. Ohya, T. Yamazaki, K. Ouchi, I. Suzuki, T. Andoh, T. Kenri, Y. Sasaki, A. Horino, M. Shintani, Y. Arakawa, and T. Sasaki. 2004. Characterization and molecular analysis of macrolideresistant Mycoplasma pneumoniae clinical isolates obtained in Japan. Antimicrob. Agents Chemother. 48:4624-4630.

34. Pereyre, S., P. Gonzalez, B. De Barbeyrac, A. Darnige, H. Renaudin, A. Charron, S. Raherison, C. Bebear, and C.M. Bebear. 2002. Mutations in 23S rRNA account for intrinsic 
resistance to macrolides in Mycoplasma hominis and Mycoplasma fermentans and for acquired resistance to macrolides in M. hominis. Antimicrob. Agents Chemother. 46: 3142-3150.

35. Schlunzen, F., R. Zarivach, J. Harms, A. Bashan, A. Tocilj, R. Albrecht, A. Yonath, and F. Franceschi. 2001. Structural basis for the interaction of antibiotics with the peptidyl transferase centre in eubacteria. Nature 413: 814-821.

36. Chantziaras, I., F. Boyen, B. Callens, and J. Dewulf. 2014. Correlation between veterinary antimicrobial use and antimicrobial resistance in food-producing animals: a report on seven countries. J. Antimicrob. Chemother. 69:827-834.

37. Barberio, A., B. Flaminio, S. De Vliegher, K. Supre, V. Kromker, C. Garbarino, N. Arrigoni, G. Zanardi, L. Bertocchi, F. Gobbo, S. Catania, and P. Moroni. 2016. Short communication: in vitro antimicrobial susceptibility of Mycoplasma bovis isolates identified in milk from dairy cattle in Belgium, Germany, and Italy. J. Dairy Sci. 99: 6578-6584.

38. Novotny, G.W., L. Jakobsen, N.M. Andersen, J. Poehlsgaard, and S. Douthwaite. 2004. Ketolide antimicrobial activity persists after disruption of interactions with do- main II of $23 \mathrm{~S}$ rRNA. Antimicrob. Agents Chemother. 48: 3677-3683.

39. Andersen, N.M., J. Poehlsgaard, R. Warrass, and S. Douthwaite. 2012. Inhibition of protein synthesis on the ribosome by tildipirosin compared with other veterinary macrolides. Antimicrob. Agents Chemother. 56:6033-6036.

40. Johansson, K.-E., M.U.K. Heldtander, and B. Pettersson. 1998. Characterization of mycoplasmas by PCR and sequence analysis with universal $16 \mathrm{~S}$ rDNA primers. In R.J. Miles and R.A.J. Nicholas (eds.), Methods in Molecular Biology, vol. 104: Mycoplasma Protocols. Humana Press Inc., Totowa, NJ, pp. 145-165.

Address correspondence to: Florence Tardy, PhD Anses Laboratoire de Lyon UMR Mycoplasmoses des Ruminants 31 Avenue Tony Garnier Lyon 69364-Cedex 07 France

E-mail: florence.tardy@anses.fr 